\title{
A CHARACTERIZATION OF CHARACTERS IN COMPLEX $m$-PSEUDOCONVEX ALGEBRAS
}

\author{
W. ŻELAZKO \\ Institute of Mathematics, Polish Academy of Sciences \\ Śniadeckich 8, P.O. Box 21, 00-956 Warszawa 10, Poland \\ E-mail: zelazko@impan.gov.pl
}

\begin{abstract}
In this paper we extend the characterization of characters given in [1], [2] and [8] onto $m$-pseudoconvex algebras. As a consequence (and a generalization) we give a characterization of continuous homomorphisms from $m$-pseudoconvex algebras into commutative semisimple $m$ pseudoconvex algebras.
\end{abstract}

1. Introduction. In the papers [1] and [2] it was shown that a subspace $M$ of codimension one in a commutative complex unital Banach algebra $A$ is a maximal ideal if and only if it consists of non-invertible elements. Equivalently: a linear functional $f$ on $A$ is multiplicative (a character) if and only if the value $f(x)$ belongs to the spectrum $\sigma(x)$ for each element $x$ in $A$. This result was extended in [8] to the non-commutative case by showing that if $f$ is a linear fuctional on a unital algebra $A$, which is multiplicative on each commutative subalgebra of $A$, then it is a character on $A$. It was already mentioned in [2] that the above result holds true for (commutative) complete locally bounded algebras and for (commutative) complete locally multiplicatively convex ( $m$-convex) algebras, the latter under an additional assumption of continuity of $f$ or closedness of $M$ (the non-commutative versions of these results follow immediately from [8]). In this paper we give a common generalization of these two results, by showing that the above-mentioned holds true for complete complex unital locally multiplicatively pseudoconvex algebras. The necessary definitions are found in the next section. The two generalizations mentioned in [2] are straightforward so that no proofs are given there. The necessary ingredient there is the existence of a submultiplicative homogeneous seminorm with respect to which the functional $f$ is continuous. In the case of a locally bounded algebra it is the spectral radius and in the case of an $m$-convex algebra it is the algebra seminorm with respect to which $f$ is continuous. In our case the only difficulty is to

2000 Mathematics Subject Classification: Primary 46H10.

The paper is in final form and no version of it will be published elsewhere. 
obtain a suitable (homogeneous) algebra seminorm. In our exposition we shall essentially follow the nice presentation of the Banach algebra result given in [5], where the result is given directly in the non-commutative case (with an argument shorter than in [8]) and is based upon a simple lemma in analytic functions of one complex variable (reproved here as Lemma 2) produced for this purpose.

As a consequence of our Theorem 1, we also give a spectral characterization of homomorphisms from $m$-pseudoconvex algebras to $m$-pseudoconvex commutative semisimple algebras (Theorem 5); it is generalization of a result obtained in [2].

2. Definitions, notations, and prerequisites. A topological algebra is a (real or complex) algebra which is a topological vector space and the multiplication is jointly continuous. A locally bounded space is a topological vector space $X$ possessing a bounded neighbourhood of zero (a set $S \subset X$ is bounded if it is absorbed by every neighbourhood of the origin, i.e. for each such neighbourhood $U$ there is a positive scalar $\lambda$ such that $\lambda S \subset U)$. A locally bounded space is necessarily metrizable and its completion is also locally bounded. The topology of a locally bounded space $X$ can be given by means of a $p$-homogeneous seminorm, $0<p \leq 1$, i.e. a non-negative subadditive fuctional $\|\cdot\|$ satisfying $\|\lambda x\|=|\lambda|^{p}\|x\|$ for all scalars $\lambda$ and all elements $x$ in $X$. Detailed information on locally bounded spaces can be found in [4]. A locally bounded algebra $A$ is a topological algebra which is a locally bounded space. Its topology can be given by means of a $p$-homogeneous algebra norm $\|\cdot\|, 0<p \leq 1$, i.e. a $p$-homogeneous norm satisfying moreover $\|x y\| \leq\|x\|\|y\|$ for all $x, y \in A$ and $\|e\|=1$, if $A$ has a unity $e$. Most facts true for Banach algebras are also true for complete locally bounded algebras (see [4], [6], [7], [9]). In particular, a commutative complete locally bounded algebra has always a continuous (non-zero) character, and so the space $\mathfrak{M}(A)$ of these characters (provided with the Gelfand topology) is non-void.

An $m$-convex algebra is a topological algebra whose topology is given by means of a family of (homogeneous) algebra seminorms, and an m-pseudoconvex algebra is a topological algebra whose topology is given by means of a family of $p$-homogeneous algebra seminorms ( $p$ depending upon the seminorm in question, $0<p \leq 1$ ). We can always assume that such a family is saturated, i.e. together with any finite set $|\cdot|{ }_{1}, \ldots,|\cdot|_{n}$ of (respectively) $p_{i}$-homogeneous seminors $\left(0<p_{i} \leq 1\right)$ the family contains its maximum given by $\|x\|=\max \left\{|x|_{i}^{p / p_{i}}: 1 \leq i \leq n\right\}$, which is a continuous $p$-homogeneous algebra norm, where $p=\min \left\{p_{1}, \ldots, p_{n}\right\}$. Under this assumption we can always claim that a continuous linear functional $f$ on the algebra $A$ in question is continuous with respect to some $p$-homogeneous seminorm $|\cdot|$ of the family considered, i.e. $|f(x)|^{p} \leq C|x|$ for some positive $C$ and all $x$ in $A$.

The completion of an $m$-convex or $m$-pseudoconvex algebra is again such an algebra. The important feature of complete $m$-pseudoconvex unital algebras is that all entire functions of one compex variable operate on them, i.e. if $\phi(\zeta)=\sum_{0}^{\infty} \alpha_{n} \zeta^{n}$ is an entire function, then for each element $x$ of the algebra $A$ in question the series $\sum_{0}^{\infty} \alpha_{n} x^{n}$ converges to an element (denoted by $\phi(x))$ in $A$. In particular, the exponential function exp operates on $A$ and for each $x$ in $A$ the element $\exp (x)$ is invertible with inverse $\exp (-x)$. 
Denote by $G(A)$ the set of all invertible elements in a unital algebra $A$ and by $\sigma_{A}(x)$ the spectrum of an element $x$ in $A$, i.e. the set $\left\{\lambda \in \mathbf{C}: x-\lambda e_{A} \notin G(A)\right.$.

Note that the class of all Banach algebras is contained in the class of all locally bounded algebras, which, in turn, is contained in the class of all $m$-pseudoconvex algebras. For more information on these algebras see [3], [6], [7], [9].

3. The results. Our main result reads as follows.

THEOREM 1. Let $A$ be a complex complete $m$-pseudoconvex algebra with a unity $e$. Then a continuous linear functional $f$ on $A$ satisfying

$$
f(e)=1
$$

is a character if and only if its kernel $f^{-1}(0)$ consists of non-invertible elements.

For the sake of completeness we give a proof of the following lemma; it is due to Walter Rudin ([5], Lemma 10.8).

LEMMA 2. Let $\varphi$ be an entire function such that

$$
\left|\exp \left[\lambda^{2} \varphi(\lambda)\right]\right| \leq \exp (|\lambda|+C)
$$

for all complex $\lambda$ and some non-negative constant $C$. Then $\varphi=0$.

Proof. Assume that $\varphi \neq 0$ and try to get a contradiction. Since $\left|\exp \left[\lambda^{2} \varphi(\lambda)\right]\right|=$ $\exp \left(\operatorname{Re}\left[\lambda^{2} \varphi(\lambda)\right]\right)$, relation (2) implies

$$
\operatorname{Re}\left[\lambda^{2} \varphi(\lambda)\right] \leq|\lambda|+C
$$

In particular

$$
\operatorname{Re}\left[\lambda^{2} \varphi(\lambda)\right] \leq 2|\lambda| \quad \text { for }|\lambda| \geq C
$$

Let $r>C$ and $C \leq|\lambda| \leq r$. By (4) we obtain for such a $\lambda$

$$
\operatorname{Re}\left[\lambda^{2} \varphi(\lambda)\right] \leq 4 r-\operatorname{Re}\left[\lambda^{2} \varphi(\lambda)\right]<4 r+m-\operatorname{Re}\left[\lambda^{2} \varphi(\lambda)\right]
$$

where $m=\max \left\{\left|\lambda^{2} \varphi(\lambda)\right|:|\lambda| \leq C\right\}$. Since $\left|\operatorname{Im}\left[\lambda^{2} \varphi(\lambda)\right]\right|=\left|\operatorname{Im}\left[4 r+m-\lambda^{2} \varphi(\lambda)\right]\right|$, the above relation implies

$$
\left|\lambda^{2} \varphi(\lambda)\right| \leq\left|4 r+m-\lambda^{2} \varphi(\lambda)\right|
$$

This relation holds, in particular, if $|\lambda|=r$, so, by the maximum principle, if $|\lambda| \leq r$. The function $4 r+m-\lambda^{2} \varphi(\lambda)$ has no zero for $|\lambda|<2 r$ (if $|\lambda| \leq C$ it follows from the definition of $m$ and if $C \leq|\lambda| \leq 2 r$ it follows from relation (5)), so the function

$$
\phi_{r}(\lambda)=\frac{r^{2} \varphi(\lambda)}{4 r+m-\lambda^{2} \varphi(\lambda)}
$$

is holomorphic for $|\lambda|<2 r$. Relation (5) implies $\left|\phi_{r}(\lambda)\right| \leq 1$ for $|\lambda|=r$ and so for all $\lambda$ satisfying $|\lambda| \leq r$. Thus for any complex $\lambda$, $\limsup _{r \rightarrow \infty}\left|\phi_{r}(\lambda)\right| \leq 1$. On the other hand, our assumption $\varphi \neq 0$ and the formula (6) imply that this limit is infinite. The contradiction shows that $\varphi(\lambda)=0$ for all complex $\lambda$ and the conclusion follows.

Proof of Theorem 1. If $f$ is a character, then the kernel $M=f^{-1}(0)$ consists of noninvertible elements. So it is sufficient to show that if $M \cap G(A)=\emptyset$, then $f$ is a character. Let $\left(|\cdot|_{\alpha}\right)$ be a saturated family of $p_{\alpha}$-homogeneous, $0<p_{\alpha} \leq 1$, algebra seminorms giving 
the topology of $A$. Since $f$ is continuous, there is an index $\alpha_{o}$ and a positive constant $C$ such that $|f(x)|^{p_{\alpha_{o}}} \leq C|x|_{\alpha_{o}}$ for all $x$ in $A$. Without loss of generality we can assume $C \geq 1$. Thus

$$
|f(x)| / C \leq 1
$$

for all $x$ in the closed unit ball $B_{o}$ for $|\cdot|_{\alpha_{o}}$, i.e. for all $x$ satisfying $|x|_{\alpha_{o}} \leq 1$. Denote by $B$ the convex envelope of $B_{o}$, i.e. the set of all convex combinations $x=\sum_{1}^{n} c_{i} x_{i}$, where $x_{i} \in B_{o}, c_{i} \geq 0$ and $\sum_{i} c_{i}=1$. For any $x$ of this form we have by (7)

$$
|f(x)| / C=\left|\sum_{i} c_{i} f\left(x_{i}\right)\right| / C \leq \sum_{i} c_{i}\left|f\left(x_{i}\right)\right| / C \leq \sum_{i} c_{i}=1,
$$

so that $|f(x)| / C \leq 1$ for all $x$ in $B$. Consequently,

$$
|f(x)| \leq C|x|_{o}
$$

for all $x$ in $A$, where $|\cdot|_{o}$ is the Minkowski fuctional for $B$, i.e. a (homogeneous) seminorm on $A$. In particular, $|\cdot|_{o}$ is not the zero seminorm. Clearly $|\cdot|_{o}$ is continuous. Since $|\cdot|_{\alpha_{o}}$ is an algebra seminorm, we have $B_{o}^{2} \subset B_{o}$, i.e. $B_{o}$ is an idempotent set. But the convex envelope of an idempotent set is again idempotent (see [3]), so that the seminorm $|\cdot|_{o}$ is submultiplicative.

Let now $x \in M$ with $|x|_{o} \leq 1$. First we are going to show that $x^{2} \in M$. Since entire functions operate on $A$, the element $\exp (\lambda x)$ is well defined for each complex $\lambda$ and it is invertible with inverse $\exp (-\lambda x)$, so that it does not belong to $M$. Put $\phi(\lambda)=f(\exp (\lambda x))$; it is a well defined complex-valued function on $\mathbf{C}$ which is non-vanishing for each complex $\lambda$. We have

$$
\phi(\lambda)=f\left(\sum_{n=0}^{\infty} \frac{\lambda^{n} x^{n}}{n !}\right)=\sum_{n} \frac{\lambda^{n} f\left(x^{n}\right)}{n !} .
$$

Since

$$
\left|f\left(x^{n}\right)\right| \leq C\left|x^{n}\right|_{o} \leq C|x|_{o}^{n} \leq C,
$$

$\phi$ is an entire function, and since it does not vanish, it is of the form $\phi(\lambda)=\exp [\psi(\lambda)]$, where $\psi$ is entire too. By (1) and (9) we have $\phi(0)=f(e)=1$ and $\phi^{\prime}(0)=f(x)=0$. This implies $\psi(0)=\psi^{\prime}(0)=0$, and so we can assume $\psi(\lambda)=\lambda^{2} \varphi(\lambda)$ with an entire $\varphi$. By (9) and (10) we have

$$
|\phi(\lambda)| \leq C \sum_{0}^{\infty}|\lambda|^{n}=C \exp (|\lambda|) .
$$

It follows that $|\psi(\lambda)|=\left|\lambda^{2} \varphi(\lambda)\right| \leq|\lambda|+\log C$ and so $\mid \exp \left[\lambda^{2} \varphi(\lambda)\right] \leq \exp \left(|\lambda|+C^{\prime}\right)$, with $C^{\prime}=\log C \geq 0$. Thus the assumption (2) of Lemma 2 is satisfied, and $\varphi=0$ by that lemma. Thus $\phi(\lambda)=1$ for all $\lambda$, and so, by the formula (9), $f\left(x^{2}\right)=0$ and $x^{2} \in M$. The same conclusion holds without assuming $|x|_{o} \leq 1$, because then we can replace $x$ by $x /|x|_{o}$. Let now $x$ be an arbitrary element of $A$. Since, by $(1), x-f(x) e \in M$, we have $x^{2}-2 f(x) x+f(x)^{2} e \in M$ and so

$$
f\left(x^{2}\right)=f(x)^{2}
$$

for all $x$ in $A$. This formula implies immediately the conclusion in the commutative case: 
just use the formula $x y=\frac{1}{2}\left[(x+y)^{2}-x^{2}-y^{2}\right]$. In the non-commutative case formula (11) gives only $f\left[(x+y)^{2}\right]=[f(x)+f(y)]^{2}$, which implies $f(x y+y x)=2 f(x) f(y)$ for all $x, y \in A$. Consequently, $f(x)=0$ implies

$$
f(x y+y x)=0
$$

for all $y$ in $A$. This fact together with formula (11) and the identity

$$
(x y-y x)^{2}=2[x(y x y)+(y x y) x]-(x y+y x)^{2}
$$

implies $f(x y-y x)=0$, which together with (12) implies $f(x y)=0$ whenever $f(x)=0$. Thus for arbitrary $x, y \in A$ we have $x-f(x) e \in M$ and so $f(x y-f(x) y)=0$, or $f(x y)=f(x) f(y)$. The conclusion follows.

In the above proof the assumption of continuity of $f$ was essential and without this assumption the result fails to be true even in the locally convex case (see [10]).

The following corollary gives a condition for existence of continuous characters for non-commutative $m$-pseudoconvex algebras.

Corollary 3. Let $A$ be a complete complex unital m-pseudoconvex algebra. Then $A$ has a continuous character if and only if $A$ has a continuous linear functional $f$ satisfying $f(x) \in \sigma(x)$ for all $x$ in $A$, or, equivalently, if and only if $A$ has a closed subspace of codimension one consisting of non-invertible elements.

REMARK 4. It is not known whether a non-commutative $m$-pseudoconvex algebra can have a discontinuous character if it has no continuous one. In fact no such complete topological algebra is known, even in the commutative case (an incomplete example is easy: the algebra of polynomials in one non-nilpotent variable contained in the radical of a Banach algebra).

We extend now Theorem 1 by replacing a functional by a linear map into a commutative semisimple $m$-pseudoconvex algebra.

Recall that a unital algebra is said to be semisimple if its Jacobson radical (the intersection of all its maximal left ideals, which is equal to the intersection of all its maximal right ideals) is the zero ideal. In the case of a commutative $m$-pseudoconvex algebra the radical is equal to the intersection of all maximal closed ideals (kernels of continuous characters), so that in such a semisimple algebra the condition $f(x)=0$ for all $f \in \mathfrak{M}(A)$ implies $x=0$.

Let $A$ and $B$ be complex algebras with unities respectively $e_{A}$ and $e_{B}$. A linear map $T: A \rightarrow B$ is said to be a unital homomorphism if it satisfies

$$
T(x y)=T x T y \quad \text { and } T e_{A}=e_{B} .
$$

The following result for commutative Banach algebras was already obtained in [2].

TheOrem 5. Let $A$ and $B$ be complete complex unital $m$-pseudoconvex algebras. Assume moreover that $B$ is commutative and semisimple. Then a continuous linear map $T$ from $A$ to $B$ is a unital homomorphism if and only if

$$
\sigma_{B}(T x) \subset \sigma_{A}(x)
$$

for all $x$ in $A$. 
Proof. If $T$ is a unital homomorphism from $A$ to $B, x \in A$ and $\lambda \notin \sigma_{A}(x)$, then $(x-$ $\left.\lambda e_{A}\right)\left(x-\lambda e_{A}\right)^{-1}=e_{A}$ and taking $T$ on both sides we obtain $\left(T x-\lambda e_{B}\right) T\left(\left[x-\lambda e_{A}\right]^{-1}\right)=$ $e_{B}$, so that $\lambda \notin \sigma_{B}(T x)$ and the formula (14) is satisfied.

Assume now that the formula (14) holds true. Since $B$ is commutative, the space $\mathfrak{M}(B)$ is non-void. For each $f$ in $\mathfrak{M}(B)$ the functional $F(x)=f(T x)$ is a continuous linear functional on $A$. Moreover

$$
F(x)=f(T x) \in \sigma_{B}(T x) \subset \sigma_{A}(x),
$$

so that the kernel of $F$ consists of non-invertible elements. By Theorem $1, F$ is a character on $A$ and so

$$
f(T(x y))=F(x y)=F(x) F(y)=f(T x) f(T y), \quad \text { or } \quad f(T(x y)-T x T y)=0
$$

for all $x, y \in A$ and all $f$ in $\mathfrak{M}(B)$. Since $B$ is semisimple, the first half of (13) follows. Similarly, $f\left(T e_{A}\right)=F\left(e_{A}\right)=1=f\left(e_{B}\right)$, or $f\left(T e_{A}-e_{B}\right)=0$ for all $f$ in $\mathfrak{M}(B)$. The second half of formula (13) and the conclusion follow now from the semisimplicity of $B$.

REMARK 6 . The above result fails to be true in case when $B$ fails to be semisimple, or fails to be commutative (even in the finite dimensional case). Let $A$ be the unital complex algebra generated by an element $t$ satisfying $t^{3}=0$. All elements of $A$ are of the form $x=\alpha(x) e+\beta(x) t+\gamma(x) t^{2}, \alpha(x), \beta(x), \gamma(x) \in \mathbf{C}$, and so $\operatorname{dim} A=3$. Put $B=A$ and define $T x=\alpha(x) e+\gamma(x) t+\beta(x) t^{2} \in B$. Clearly $\sigma_{B}(T x)=\{\alpha(x)\}=\sigma_{A}(x)$, but $T$ is not a homomorphism since $T(t)^{2}=0$ and $T\left(t^{2}\right)=t$.

Put now $B=M_{3}$, the algebra of all $3 \times 3$ complex matrices. Let $J_{i}, i=1,2,3$, be the set of all matrices in $B$ with all entries in the $i$-th column equal to 0 . It can be easily seen that for each $i$ the set $J_{i}$ is a left ideal in $B$. It is a maximal left ideal, since for each matrix $x \notin J_{i}$ we can find a matrix $y \in J_{i}$ with $\operatorname{det}(x+y) \neq 0$, so that $x+y$ is invertible in $B$. Thus $B$ is semisimple, since $J_{1} \cap J_{2} \cap J_{3}=\{0\}$. By the left regular representation we can imbed our previous algebra $A$ in $B$ as a unital subalgebra. This imbedding is given by

$$
x \rightarrow\left(\begin{array}{ccc}
\alpha(x) & \beta(x) & \gamma(x) \\
0 & \alpha(x) & \beta(x) \\
0 & 0 & \alpha(x)
\end{array}\right),
$$

so that the previously considered map $T$ can be treated as a map from $A$ into $B$, and $B$ is this time semisimple. As before, we have $\sigma(T x)=\sigma(x)$ but $T$ is not a homomorphism.

Note that the last construction is rather formal, since the algebra $T(A)$ is not semisimple. The author does not know what happens if instead of assuming the semisimplicity of $B$ we assume only the semisimplicity of the closure of $T(A)$ in $B$.

\section{References}

[1] A. Gleason, A characterization of maximal ideals, J. Analyse Math. 19 (1967), 171-172.

[2] J.-P. Kahane and W. Żelazko, A characterization of maximal ideals in commutative Banach algebras, Studia Math. 29 (1968), 339-343.

[3] E. Michael, Locally multiplicatively-convex topological algebras, Mem. Amer. Math. Soc. 11 (1952). 
[4] S. Rolewicz, Metric Linear Spaces, PWN, Warszawa, 1972.

[5] W. Rudin, Functional Analysis, McGraw-Hill, New York, 1973.

[6] W. Żelazko, On the locally bounded and m-convex topological algebras, Studia Math. 19 (1960), 333-356.

[7] W. Żelazko, Metric Generalizations of Banach Algebras, Dissertationes Math. [Rozprawy Mat.] 47, 1965.

[8] W. Żelazko, A characterization of multiplicative-linear functionals in complex Banach algebras, Studia Math. 30 (1968), 159-161.

[9] W. Żelazko, Selected Topics in Topological Algebras, Aarhus Univ. Lecture Notes Series No 31, 1971.

[10] W. Żelazko, On multiplicative-linear functionals, Colloq. Math. 28 (1973), 251-255. 